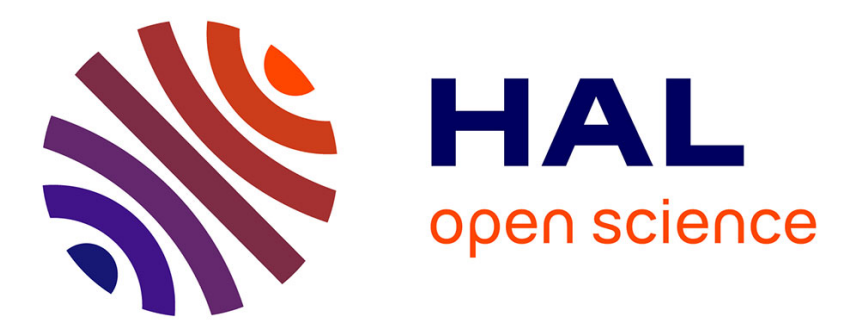

\title{
Magnetism tailored by mechanical strain engineering in PrVO 3 thin films
}

\author{
D. Kumar, A. David, A. Fouchet, A. Pautrat, J. Varignon, C. Jung, U. \\ Lüders, B. Domengès, O. Copie, P. Ghosez, et al.
}

\section{- To cite this version:}

D. Kumar, A. David, A. Fouchet, A. Pautrat, J. Varignon, et al.. Magnetism tailored by mechanical strain engineering in PrVO 3 thin films. Physical Review B, 2019, 99 (22), pp.224405. 10.1103/PhysRevB.99.224405 . hal-03014268

\section{HAL Id: hal-03014268 \\ https://hal.science/hal-03014268}

Submitted on 20 Nov 2020

HAL is a multi-disciplinary open access archive for the deposit and dissemination of scientific research documents, whether they are published or not. The documents may come from teaching and research institutions in France or abroad, or from public or private research centers.
L'archive ouverte pluridisciplinaire HAL, est destinée au dépôt et à la diffusion de documents scientifiques de niveau recherche, publiés ou non, émanant des établissements d'enseignement et de recherche français ou étrangers, des laboratoires publics ou privés. 


\title{
Magnetism tailored by mechanical strain engineering in $\mathrm{PrVO}_{3}$ thin films
}

\author{
D. Kumar, ${ }^{1}$ A. David, ${ }^{1}$ A. Fouchet, ${ }^{1}$ A. Pautrat,${ }^{1}$ J. Varignon, ${ }^{2}$ C. U. Jung, ${ }^{3}$ U. Lüders, ${ }^{1}$ B. Domengès,${ }^{1}$ \\ O. Copie, ${ }^{4}$ P. Ghosez, ${ }^{2}$ and W. Prellier ${ }^{1, *}$ \\ ${ }^{1}$ Laboratoire CRISMAT, CNRS UMR 6508, ENSICAEN, Normandie Université, 6 Bd Maréchal Juin, F-14050 Caen Cedex 4, France \\ ${ }^{2}$ Physique Théorique des Matériaux, Université de Liège (B5), B-4000 Liège, Belgium \\ ${ }^{3}$ Department of Physics, Hankuk University of Foreign Studies, Yongin, Gyeonggi 17035, Korea \\ ${ }^{4}$ Institut Jean Lamour, CNRS-Université de Lorraine, Campus Artem, 2 allée André Guinier BP 50840, 54011 Nancy cedex, France
}

(Received 14 February 2019; published 3 June 2019)

\begin{abstract}
Transition-metal oxides with an $\mathrm{ABO}_{3}$ perovskite structure exhibit strongly entangled structural and electronic degrees of freedom and thus one expects to unveil exotic phases and properties by acting on the lattice through various external stimuli. Using the Jahn-Teller active praseodymium vanadate $\operatorname{Pr}^{3+} \mathrm{V}^{3+} \mathrm{O}_{3}$ compound as a model system, we show that $\mathrm{PrVO}_{3}$ Néel temperature $T_{N}$ can be raised by $40 \mathrm{~K}$ with respect to the bulk when grown as thin films. Using advanced experimental techniques, this enhancement is unambiguously ascribed to a tetragonality resulting from the epitaxial compressive strain experienced by the films. First-principles simulations not only confirm experimental results, but they also reveal that the strain promotes an unprecedented orbital ordering of the $\mathrm{V}^{3+} d$ electrons, strongly favoring antiferromagnetic interactions. These results show that an accurate control of structural aspects of oxides is the key for unveiling unexpected phases in oxides.
\end{abstract}

DOI: 10.1103/PhysRevB.99.224405

\section{INTRODUCTION}

Transition metal oxides with an $\mathrm{ABO}_{3}$ perovskite structure are multifunctional materials displaying a large collection of properties such as ferroelectricity, metal-to-insulator transition, high $T_{C}$ superconductivity, and colossal magnetoresistance (CMR), for instance [1-4]. This richness of physical behaviors emerges through strongly coupled structural, electronic, and magnetic degrees of freedom, enabling possibilities to control the material's properties with external stimuli [5]. Among all approaches, strain engineering allowed by minute deposition of oxides as thin films on a range of commercially available substrates is likely the most adopted strategy to unveil hidden phases in bulk. Most striking examples achieved with strain engineering are (i) the observation of ferroelectricity in $\mathrm{SrTiO}_{3}$ films under tensile epitaxial strain [6], an otherwise quantum paralectric compound in bulk, (ii) the rich ferroelectric phase diagram of $\mathrm{BiFeO}_{3}$ as a function of the applied epitaxial strain [7], or (iii) the control of magnetoresistive properties in $R_{1-x} A_{x} \mathrm{MnO}_{3}$ films ( $R=$ rare earth, $A=\mathrm{Ca}, \mathrm{Sr}$ ) [8,9].

In the search of multifunctional materials with possibly unprecedented properties, one must consider materials with nearly degenerate ground states that could be tailored by epitaxial strain. Along with the widely studied rare-earth manganites [10-12], rare-earth vanadate perovskites $R \mathrm{VO}_{3}(R=$ $\mathrm{Lu}-\mathrm{La}, \mathrm{Y})$ are prototypical compounds showing strongly coupled structural-spin-orbital properties [13,14]. At high temperature, $R \mathrm{VO}_{3}$ compounds are paramagnetic insulators adopting the usual orthorhombic Pbnm symmetry displayed by perovskites and characterized by $a^{-} a^{-} c^{+}$octahedral

\footnotetext{
*wilfrid.prellier@ensicaen.fr
}

rotations. Due to the intrinsic instability displayed by the $\mathrm{V}^{3+} t_{2 g}^{2}$ electronic configuration [15], a Jahn-Teller distortion appears and induces a symmetry lowering to a monoclinic $P 2_{1} / b$ structure at the temperature $T_{o o}$. It produces a G-type orbital ordering with alternating occupancy of the $d_{x z}$ and $d_{y z}$ orbitals on neighboring $\mathrm{V}$ sites according to a rocksalt-like pattern - the second electron is located in the low energy $d_{x y}$ orbital on all $\mathrm{V}$ sites. It is then followed by a magnetic transition at $T_{N}<T_{o o}$-except for $\mathrm{LaVO}_{3}$ for which $T_{N}$ is $2 \mathrm{~K}$ above $T_{o o}$ [16] - to a C-type AFM order explained by Kugel-Khomskii and Goodenough-Kanamori rules [17,18]. Finally, for vanadates involving rare earth with a small ionic radius $(R=\mathrm{Lu}-\mathrm{Dy}, \mathrm{Y})$, the compound goes back to an orthorhombic Pbnm symmetry characterized by an alternative Jahn-Teller distortion producing a C-type orbital arrangement of $t_{2 g}$ orbitals-columnar arrangement along the $c$ axis of alternating $d_{x z}$ and $d_{y z}$ orbitals-that is associated with a G-type AFM order at $T_{N 2}$.

It is obvious that the chemical pressure induced by $A$-site cations dramatically influences the electronic and magnetic states of the vanadates. Likewise, external stimuli such as hydrostatic pressure or partial $A$ site substitution can also tune the material properties $[19,20]$. Regarding thin films, a precise control of oxygen vacancies concentration in $\mathrm{PrVO}_{3}$ grown on a $\mathrm{SrTiO}_{3}$ substrate was recently shown to produce a substantial chemical strain, offering a pathway to modify the Néel temperature on a range of $30 \mathrm{~K}$ using a unique substrate type [21]. Nevertheless, basic questions remain largely unexplored in these compounds: can we tune the vanadate properties using various epitaxial strains, and eventually promote new electronic phases? Aiming at providing answers to these important questions, we have studied the effect of epitaxial strains on the praseodymium vanadate perovskite using advanced experimental techniques. We show that the 
Néel temperature can be continuously raised by $40 \mathrm{~K}$ with respect to the bulk by increasing the compressive epitaxial strain. Our first-principles simulations confirm the experimentally observed trend for $T_{N}$, but amazingly they also reveal that this strong enhancement is associated with an unprecedented orbital order of $t_{2 g}$ levels.

\section{METHODS}

Experiments. $\mathrm{PrVO}_{3}(\mathrm{PVO})$ thin films $(t \sim 50 \mathrm{~nm})$ were grown on various substrates such as (110)- $\mathrm{YAlO}_{3}(\mathrm{YAO})$, (100)- $\mathrm{LaAlO}_{3}$ (LAO), (100)-( $\left.\mathrm{La}, \mathrm{Sr}\right)(\mathrm{Al}, \mathrm{Ta}) \mathrm{O}_{3}$ (LSAT), and (100)- $\mathrm{SrTiO}_{3}$ (STO) using the pulsed laser deposition (PLD) method. A KrF excimer laser $(\lambda=248 \mathrm{~nm})$ with repetition rate of $2 \mathrm{~Hz}$ and laser fluence of $\sim 2 \mathrm{~J} / \mathrm{cm}^{2}$ was focused on stoichiometric ceramic targets. All the films used in this study were deposited at an optimum growth temperature $\left(T_{G}\right)$ of $650^{\circ} \mathrm{C}$ and under oxygen partial pressure $\left(P_{\mathrm{O}_{2}}\right)$ of $10^{-6}$ mbar. The thickness of PVO films was kept nearly constant at $50 \mathrm{~nm}$. To identify the lattice mismatch, the pseudocubic lattice parameters of YAO, LAO, LSAT, and STO were used as $3.700 \AA, 3.790 \AA, 3.868 \AA$, and $3.905 \AA$, respectively. The crystallinity and the structure were characterized using conventional high resolution x-ray diffraction (XRD) technique (Bruker D8 Discover diffractometer, $\mathrm{Cu} K_{\alpha 1}$ radiation, $\lambda=1.54056 \AA$ ). The surface morphology was investigated using atomic force microscopy (AFM) PicoSPM. The resistivity $[\rho(T)]$ measurements were performed using the four point probe technique in a quantum design physical properties measurement system (PPMS). The magnetic measurements were obtained using superconducting quantum interface device magnetometer (SQUID), as a function of temperature $(T)$ and magnetic field $(H)$. Transmission electron microscopy (TEM)-scanning transmission electron microscopy (STEM) study was carried out on a JEM-ARM200F, operating at $200 \mathrm{kV}$, equipped with a cold field emission gun and double TEM-STEM Cs correctors, ensuring lattice TEM or STEM image resolution below $0.1 \mathrm{~nm}$, and JEOL EDS system. Thin TEM lamellae were prepared in a dual-beam system (FEIHELIOS 600) equipped with easy-lift manipulator designed for in situ lift-out thin lamella preparations.

Theoretical calculations. First principles calculations are performed using density functional theory with the VASP package $[22,23]$. We have employed the PBEsol functional in addition to a $\mathrm{U}$ potential on $\mathrm{V} d$ levels of $3.5 \mathrm{eV}$, entering as a single effective parameter [24], in order to better cancel the spurious self-interaction term. This parameter was fitted in Refs. [21,25] and was providing correct electronic, magnetic, and structural features for the $\mathrm{PrVO}_{3}$ ground state. $\operatorname{Pr} 4 f$ electrons are not considered in the study and are included in the projected augmented wave (PAW) [26] potential. Unit cells used in our simulations correspond to a $(2 a, 2 a, 2 a)$ cubic cell allowing for the oxygen cage rotations and Jahn-Teller motions to develop (i.e., eight formula units). The energy cutoff is set to $500 \mathrm{eV}$ and a $4 \times 4 \times 4 k$-point mesh is employed. Four magnetic states are explored in our simulations, namely the C, G, and A-type SO as well as a ferromagnetic solution. We have considered two growth orientations for the films with the in-phase rotation axis [i.e., the (001) Pbnm axis] lying either along the substrate or perpendicular to it. We then block two $\mathrm{PrVO}_{3}$ lattice parameters to those of the substrate and relax the magnitude of the remaining lattice parameter, although restricting it to be orthogonal to the substrate due to the presence of $90^{\circ}$ oriented domains [21].

Nearest neighbor magnetic exchange integrals $J_{1}$ and $J_{2}$, corresponding to interactions along the (110) [or (1-10)] and (001) directions, respectively, are extracted by mapping energies of FM, A, C, and G-type spin orderings on a Heisenberg model of the form $\hat{H}=-J \sum_{i<j} S_{i} \cdot S_{j}$, where the sum runs over all possible sites $i$ and $j$ in the cell. The cell is fixed to the ground state structure for each strain value. In order to avoid modifications of the electronic structure due strongly entangled spin-orbital degrees of freedom in $\mathrm{PrVO}_{3}$ [17], we have frozen the $d$ orbital occupancies to that of the lowest energy state using the modified DFT $+U$ routine of VASP [27] and we simply switched spin channels to account for the magnetic order. The Néel temperature is then computed using a mean-field model with $T_{N} \propto\left(2 J_{1}+J_{2}\right)$.

\section{RESULTS AND DISCUSSION}

We have grown a series of $\mathrm{PrVO}_{3}(\mathrm{PVO})$ thin films using pulsed laser deposition on (001)-oriented substrates a priori yielding either nearly no epitaxial strain $\left[\mathrm{SrTiO}_{3}(\mathrm{STO})\right.$ substrate] or compressive strain $\left[(\mathrm{La}, \mathrm{Sr})(\mathrm{Al}, \mathrm{Ta}) \mathrm{O}_{3}(\mathrm{LSAT})\right.$, $\mathrm{LaAlO}_{3}(\mathrm{LAO})$, and $\mathrm{YAlO}_{3}(\mathrm{YAO})$ substrates] with respect to the bulk PVO [see Fig. 1(a)].

Figure 1(b) displays $\theta-2 \theta$ scan for the epitaxially grown PVO thin films. For most of the substrates, clear thickness fringes are observed around the main diffraction peaks, confirming a uniform thickness and smooth interfaces of the films. The film thickness estimated using these fringes in the diffraction pattern is actually around $50 \mathrm{~nm}$ for all films leading to a growth rate $(\sim 0.09 \AA /$ pulse $)$. In the case of the LAO substrate, these oscillations are however small and subtle, probably due to the presence of twin domains in the LAO substrate [28]. The film's surfaces are quite smooth, presenting clear steps and terraces [see inset of Fig. 1(b)]. For example, the RMS surface roughness of the PVO/LAO film was found around $2.3 \AA$, indicating a flat surface. The evolution of the out-of-plane lattice parameter (calculated from XRD data) is plotted as a function of the substrate lattice parameter in Fig. 1(c). Surprisingly, it presents a maximum for the LAO substrate and a relatively lower lattice parameter for $\mathrm{PVO} / \mathrm{YAO}$ film. This indicates the ability of PVO/LAO film to adopt large strain and a lower or no strain in PVO/YAO film, which is anticipated for such a large lattice mismatch.

To identify the strain states, reciprocal space maps were recorded around $(103)_{c}$ (where the index $c$ refers to the cubic perovskite sublattice) planes of LAO, LSAT, and STO and (212) plane of YAO [Fig. 1(d)]. The X-ray reciprocal space mapping shows well-developed film peaks in the lower region and strong substrate peaks in the upper region for all the PVO films. Since the horizontal peak positions of the PVO film coincide with those of the substrate for both LSAT and STO, we deduce that the film is fully strained with the substrate and has the same in-plane lattice constant. In the case of LAO, the small shift of the film peak to lower $Q_{\text {in }}$ value suggests an increase of the in-plane lattice parameter and a partially relaxed film, which confirms a flexibility of the 

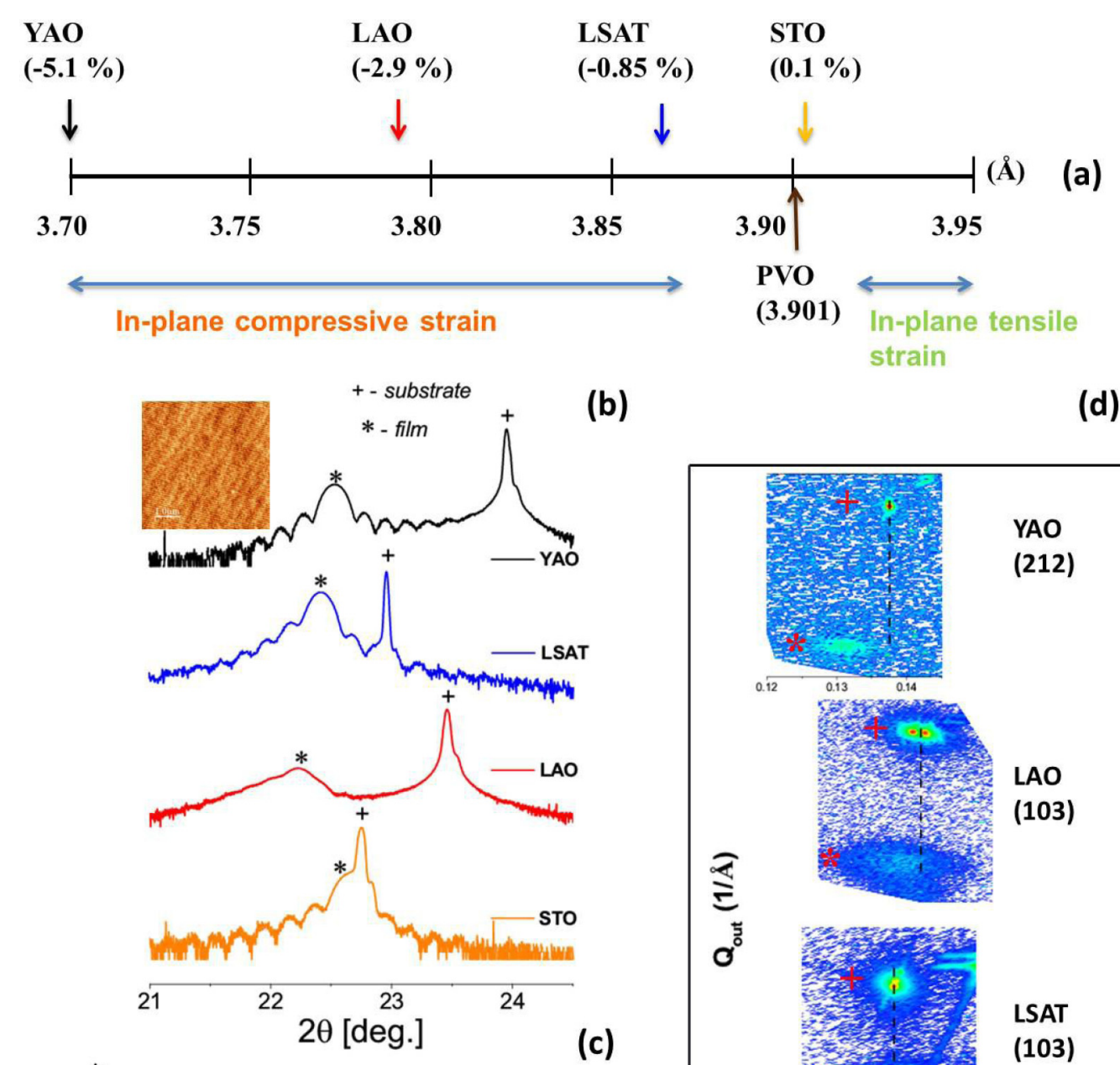

(b)

(d)
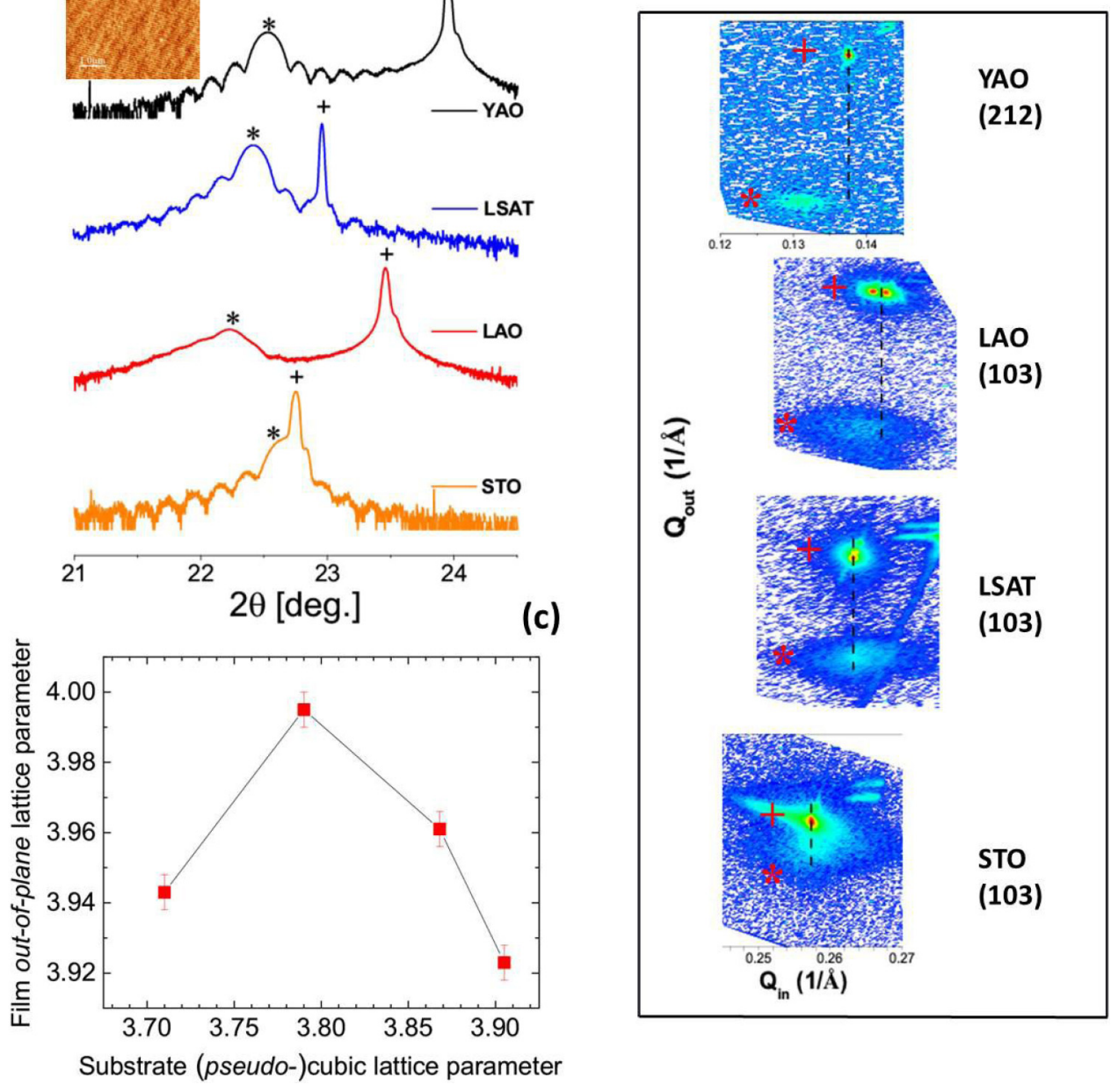

FIG. 1. (a) Lattice mismatch between bulk stoichiometric $\mathrm{PrVO}_{3}$ and the various substrates at room temperature. (b) $\theta-2 \theta$ HRXRD scan for a series of $50 \mathrm{~nm}$ thick PVO thin films on different substrates. (001) $)_{C}$ diffraction peak of the PVO film and substrate (LSAT, LAO, STO) and $(110)_{c}$ of YAO substrate are indicated by $*$ and + , respectively. The inset of (b) is a $5 \mu \mathrm{m} \times 5 \mu \mathrm{m}$ AFM image of PVO film deposited on LAO substrate (RMS surface roughness $\sim 2.3 \AA$ ). (c) Evolution of the out-of-plane lattice parameter as a function of the substrate pseudocubic lattice parameter. (d) RSMs around pseudocubic (103) plane of STO, LAO, and LSAT and (212) plane of YAO substrate. The substrate peaks are sharp, intense, shown by plus $(+)$ sign, and located on the upper region of RSM image. The film peaks [shown by asterisks $(*)$ ] are broader and located on the lower region of the image. The solid and dotted lines are only guides to the eyes.

PVO structure for a large strain associated with large lattice mismatch of $-2.9 \%$. Finally, we see that the PVO film is fully relaxed on YAO, indicating that the growth is not coherent for this peculiar substrate, which can be explained by large compressive lattice mismatch $(-5.1 \%)$. Additionally, the film relaxes in order to minimize the accumulated strain energy [28-30].
Moreover, the out-of-plane lattice parameter is well above the bulk value (3.901 ̊) for all PVO films irrespective of the strain (compressive/tensile). This is due to low oxygen partial pressures used during the growth which induces oxygen vacancies in the film, resulting in an enhancement of lattice parameter [31,32]. Nevertheless, with increase of the in-plane compressive strain, the out-of plane lattice parameter 
TABLE I. Summary of observed lattice parameters, experimental lattice mismatch, pseudocubic cell volumes, distortion (ratio of the out-of-plane to in-plane PVO lattice parameter), residual strain calculated out-of-plane, i.e., $\epsilon_{110}$, Néel temperature $\left(T_{N}\right)$, and coercivity $\left(H_{c}\right)$ of PVO films.

\begin{tabular}{|c|c|c|c|c|c|c|c|c|c|}
\hline Substrate & $\begin{array}{c}\text { In-plane lattice } \\
\text { parameter of } \\
\text { substrate }(\AA)\end{array}$ & $\begin{array}{l}\text { Lattice } \\
\text { mismatch } \\
(\%)\end{array}$ & $\begin{array}{l}\text { In-plane lattice } \\
\text { parameter of } \\
\text { film }(\AA)\end{array}$ & $\begin{array}{c}\text { Out-of- } \\
\text { plane lattice } \\
\text { parameter of } \\
\text { film }(\AA)\end{array}$ & $\begin{array}{l}\text { Pseudo-cubic } \\
\text { unit cell } \\
\text { volume }\left(\AA^{3}\right)\end{array}$ & Distortion & $\begin{array}{c}\text { Residual } \\
\text { strain (out of } \\
\text { plane) }(\%)\end{array}$ & $T_{N}(\mathrm{~K})$ & $H_{c}(\mathrm{~T})$ \\
\hline YAO & 3.710 & -5.15 & 3.860 & 3.943 & 58.75 & 1.022 & 1.077 & 134 & 2.40 \\
\hline LAO & 3.790 & -2.93 & 3.830 & 3.995 & 58.60 & 1.043 & 2.412 & 172 & 3.25 \\
\hline LSAT & 3.868 & -0.85 & 3.868 & 3.961 & 59.26 & 1.024 & 1.540 & 125 & 2.70 \\
\hline
\end{tabular}

is enhanced as expected when going from the LSAT to LAO substrate. The out-of-plane lattice parameter of PVO/YAO film is however much smaller, which is in agreement with a relaxed film as shown in Fig. 1(d).

The residual strain was calculated using $\epsilon_{h k l}=$ $\left(d_{h k l}^{0}-d_{h k l}\right) / d_{h k l}^{0}$, where $d_{h k l}^{0}$ and $d_{h k l}$ are the pseudocubic PVO bulk and film out-of-plane lattice parameters, respectively. Interestingly, across the series, the measured strain increases from $\sim 0.5 \%$ for $\mathrm{PVO} / \mathrm{STO}$ to $\sim 2.4 \%$ for $\mathrm{PVO} /$ LAO (Table I).

Table II details the possible expansion of unit cell due to the presence of oxygen vacancies. The film's out-of-plane lattice parameter $\left[a_{\perp}\right.$ (expected) $]$ and epitaxial lattice mismatch $\left(\epsilon_{\|}\right)$ are linked by the Poisson ratio (v) as follows [33]:

$$
a_{\perp}=\left[1-\frac{2 v}{1-v} \cdot \epsilon_{\|}\right] a_{0},
$$

where $a_{0}$ is the film's unstrained lattice parameter. Indeed, from Table II, we observe that, as tensile strain increases, the volume of the unit cell expands. This is essentially observed in the case of the STO substrate, where the out-of-plane lattice parameter increases by $0.7 \%$ due to the presence of oxygen vacancies, as compared to the expected one. On the contrary, for compressive strain, the cell volume is nearly equal to the bulk one $\left(\mathrm{V}_{p c} \sim 59.36 \AA^{3}\right)$, suggesting the absence of oxygen vacancies as reported in $\mathrm{CaMnO}_{3}$ and $\mathrm{SrMnO}_{3}$ films [34,35], and confirming that the observed effect results mainly from epitaxial strain. Finally, for the LSAT substrate, the cell volume is close to the bulk one and the out-of-plane lattice parameter is slightly higher than the expected one. This establishes the LSAT substrate in an intermediate regime, where the strain is mainly dominated by the epitaxial one with a small amount of oxygen vacancies.

In order to obtain details of the microstructure, transmission electron microscopy (TEM) studies were performed on cross-sectionnal thin lamellae prepared for each sample. The lamellae were oriented in order to observe both the outof-plane axis, i.e., growth direction, and one in-plane axis, characteristic of the perovskite structure. The TEM study, through electron diffraction (ED), high resolution TEM, and scanning-TEM imaging allowed a local characterization of the PVO films, in terms of orientation with respect to the substrate, evolution of the parameters (strain), nanostructure (domains), and quality of the film-substrate interface [36], and more details can be found elsewhere [37]. The observed thickness of the PVO films is close to those calculated from XRD around $50 \mathrm{~nm}$. The selected area electron diffraction (SAED) study is in complete agreement with $\mathrm{x}$-ray reciprocal space mapping. Almost no strain is observed on the STO subtrate with a perfect adequation of in- and out-of-plane lattice parameters (deduced from a perfect superposition of diffraction spots of substrate and film). In the case of the YAO substrate, two electron diffraction patterns can be clearly distinguished, one exhibiting YAO parameters and the second related to PVO parameters, along both in- and out-of-plane directions [36]. Thus there is almost no interaction between the YAO substrate and PVO film.

On LSAT and LAO substrates, both parameters are influenced: the strain being compressive, the PVO in-plane lattice parameter is decreased to fit the one of the substrate, leading to an increase of the out-of-plane lattice parameter. The PVO films always exhibit small domains (several tens of nanometers) [36]. In most of the observations, the PVO

TABLE II. Quantification of possible oxygen vacancies in tensile and compressive strain. The estimation of $a_{\perp}$ (expected) is established based on the lattice mismatch and Poisson ratio of PVO, $v \sim 0.39$.

\begin{tabular}{|c|c|c|c|c|c|}
\hline Substrate & $\begin{array}{l}\text { Lattice } \\
\text { mismatch } \\
(\%)\end{array}$ & $\begin{array}{c}a_{\perp} \\
\text { (expected) } \\
(\AA)\end{array}$ & $\begin{array}{c}a_{\perp} \\
\text { (actual) } \\
(\AA)\end{array}$ & $\begin{array}{c}\text { Expansion } \\
\text { due to } \mathrm{O} \\
\text { vacancies }(\%)\end{array}$ & $\begin{array}{c}V_{u c} \text { (present } \\
\text { expt. work) } \\
\left(\AA^{3}\right)\end{array}$ \\
\hline LSAT & -0.85 & 3.950 & 3.961 & 0.27 & 59.26 \\
\hline LAO & -2.93 & 4.047 & 3.995 & -1.28 & 58.60 \\
\hline YAO & -5.15 & 4.157 & 3.943 & -5.14 & 58.75 \\
\hline
\end{tabular}




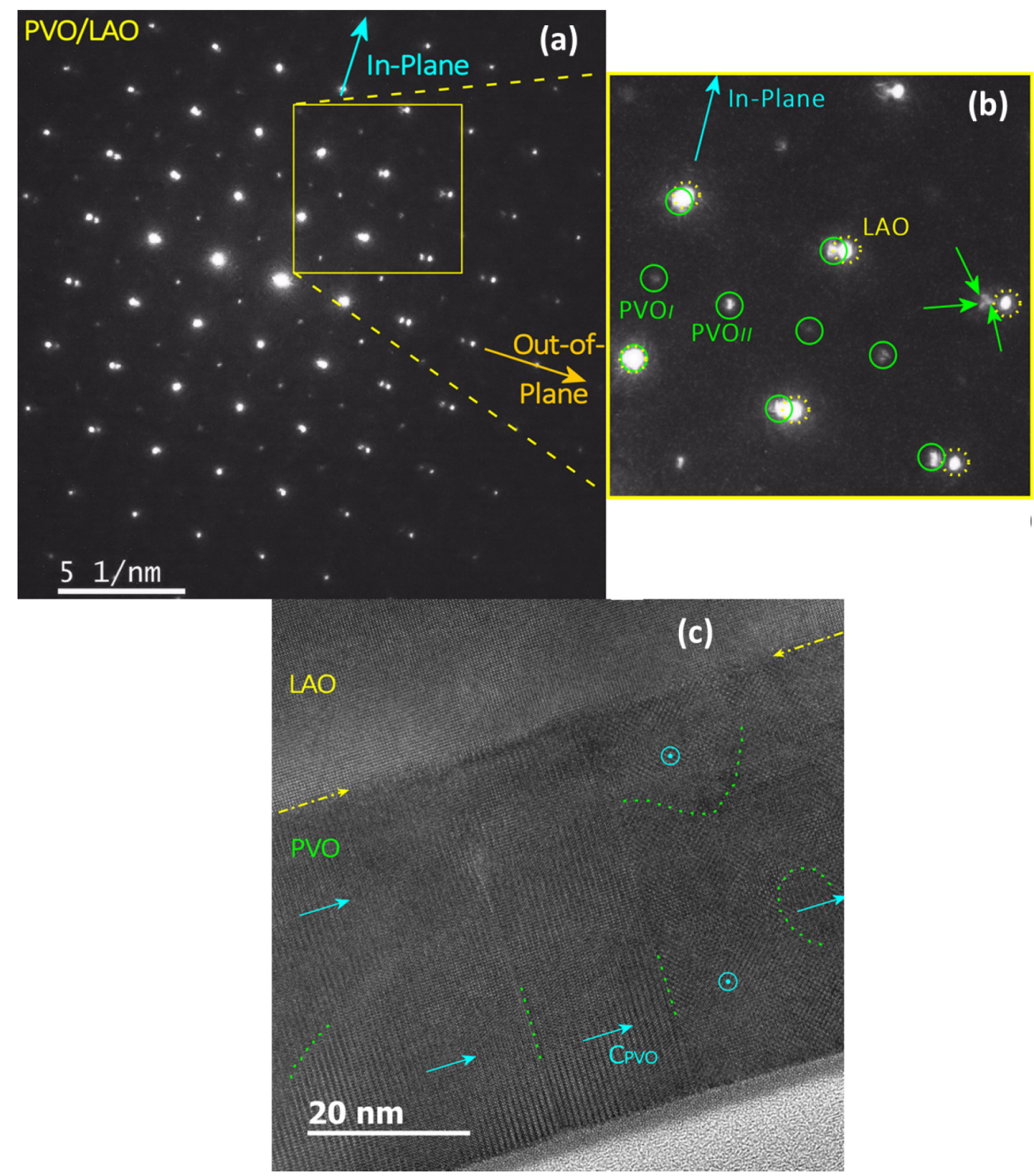

FIG. 2. (a) Typical SAED pattern of PVO/LAO sample; several patterns can be distinguished, especially along out-of-plane direction. (b) Enlargement showing the complex splitting of PVO reflections (green arrows). (c) HRTEM image of PVO film on LAO substrate. The orientation of $\mathrm{c}_{\mathrm{PVO}}$ is given on several domains. It always lies in plane, either parallel (cyan solid arrows) or perpendicular (dotted circle) to the lamella plane. Domains are outlined with dotted lines. The interface between LAO substrate and PVO film exhibits contrast perturbation indicative of strains, depicted by dashed arrows.

$[001]_{o}$ lies in plane, and the diffraction spots related to $2 \times a_{p c}$ along the growth direction are either weak (STO, YAO) or nonexistent (LAO).

The SAED pattern shown in Figs. 2(a) and 2(b) illustrates these observations for PVO film grown on the LAO substrate: several patterns are superimposed, one LAO and two PVO ones. The latter correspond to several diffracting domains [labeled $I$ and $I I$ in Fig. 2(b)] having the [110] reciprocal axis out of plane. Moreover, the enlargement of SAED pattern shows a more complex splitting of PVO reflections that could be due to deformation of the PVO framework from one domain to the other. The domain size was evaluated from several TEM images, covering about $0.5 \mu \mathrm{m}$ of the PVO film. It appeared that despite an apparent columnar growth, several domains may be observed from the bottom to the surface of the film [Fig. 2(c)]. In addition, measurements suggest that domains are smaller in size when the PVO film is not strained (on STO and YAO substrates). Stacking faults were observed in the upper part of the PVO film, on about $1 / 4$ of the thickness and usually extend parallel to the growth direction. They involve either the oxygen framework or both oxygen and cation ones.

To investigate the effect of biaxial strain on the physical properties, the transport properties $[\rho(T)]$ of PVO films were investigated [36]. The insulatorlike $\rho(T)$ behavior was observed for PVO films on LAO, LSAT, and YAO. On the contrary, the PVO/STO film displayed a conductinglike behavior, which is likely resulting from the presence of the oxygen vacancies in the STO substrate [31,32,38,39]. 

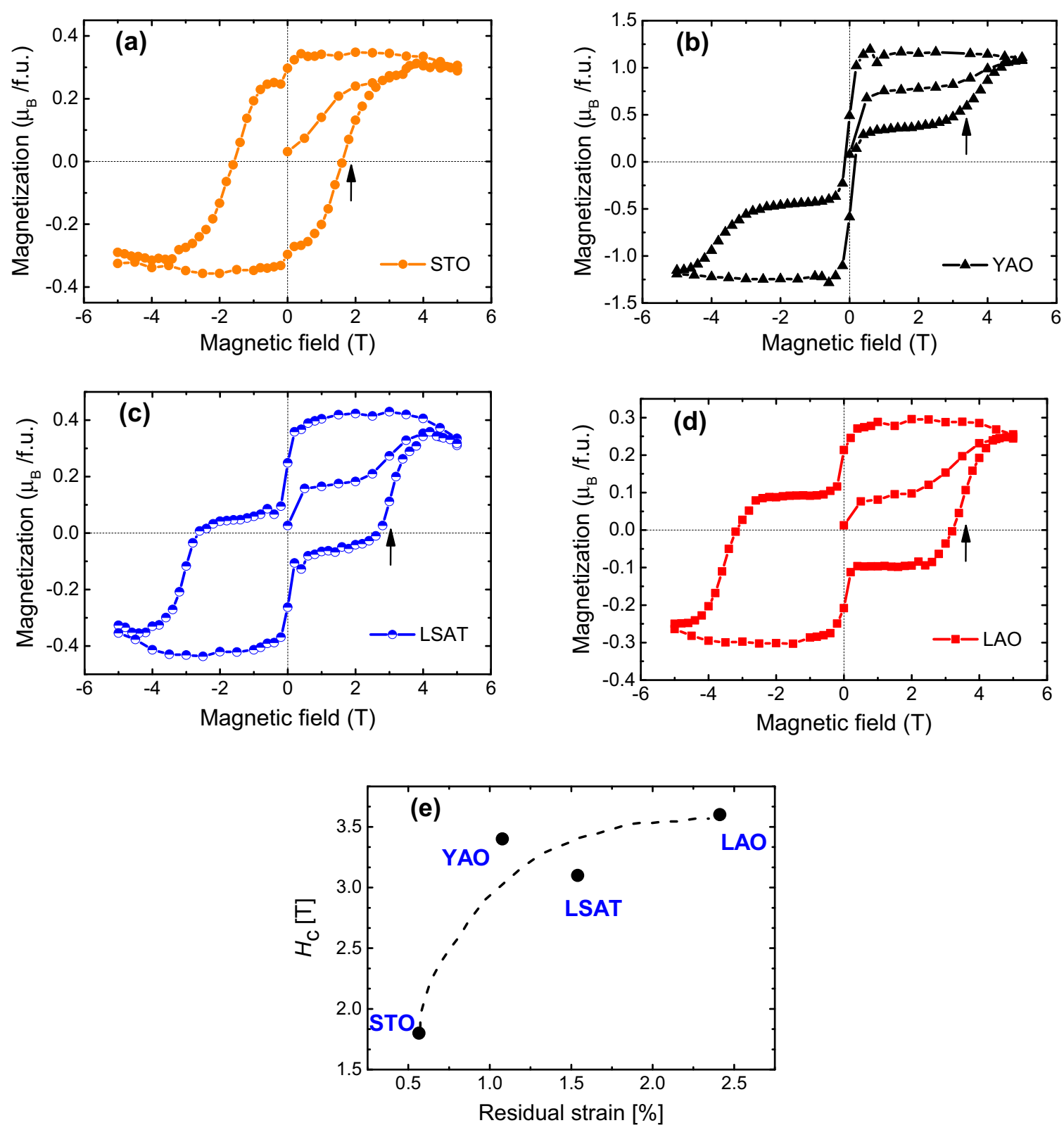

FIG. 3. Magnetic measurement as a function of in-plane applied magnetic field for PVO films grown on (a) STO, (b) YAO, (c) LSAT, and (d) LAO substrate performed at $20 \mathrm{~K}$. The arrow in the above figures represents the hard ferromagnetic component, based on which the coercive field was calculated. (e) Evolution of coercive field with residual strain. The dashed line is only a guide to the eyes.

To examine the effect of the biaxial strain on the magnetic properties of PVO films, the magnetization $(M)$ of PVO films was measured as a function of the in-plane applied magnetic field $(H)$ and temperature $(T)$ (Figs. 3 and 4).

At low $T$, all PVO films show a small magnetization with a hysteresis loop indicating two magnetic phases, a soft and a hard one [Figs. 3(a)-3(d)]. For instance, for PVO/STO, the soft contribution shows a coercive field $H_{c}$ at $\sim 0.2 \mathrm{~T}$ and the hard one at $\sim 1.8 \mathrm{~T}$. While Copie et al. already observed a soft ferromagnetic behavior for the bulk PVO (our case) with $H_{c} \sim 0.019 \mathrm{~T}$ [21], a hard ferromagnetic behavior was also reported for bulk PVO in Refs. [40,41]. This discrepancy of coercivity between bulk and PVO films could be explained by the microstructure. The presence of different variants of the PVO orthorhombic cell (see TEM section) induces different pinning centers, and thus increases the energy to return the magnetization, similar to what is observed in the orthoferrite $\mathrm{YFeO}_{3}$ [42]. Furthermore, it is interesting to note that the weightage of soft and hard magnetic phases can be modified by interplaying the epitaxial strain. For instance, hard and soft components were evaluated as $\Delta M_{\text {hard }}=$ $85 \%$ and $\Delta M_{\text {soft }}=15 \%$ for PVO $/ \mathrm{STO}, \Delta M_{\text {hard }}=70 \%$ and $\Delta M_{\text {soft }}=30 \%$ for PVO/LAO, $\Delta M_{\text {hard }}=60 \%$ and $\Delta M_{\text {soft }}=$ $40 \%$ for PVO/LSAT, and $\Delta M_{\mathrm{hard}}=45 \%$ and $\Delta M_{\mathrm{soft}}=55 \%$ for PVO/YAO [Figs. 3(a)-3(d)], as in Refs. [36,42]. In addition, the fact that PVO/YAO film has a large percentage of soft component is interpreted as a behavior similar to the bulk PVO, since the film is fully relaxed (as shown by XRD and 

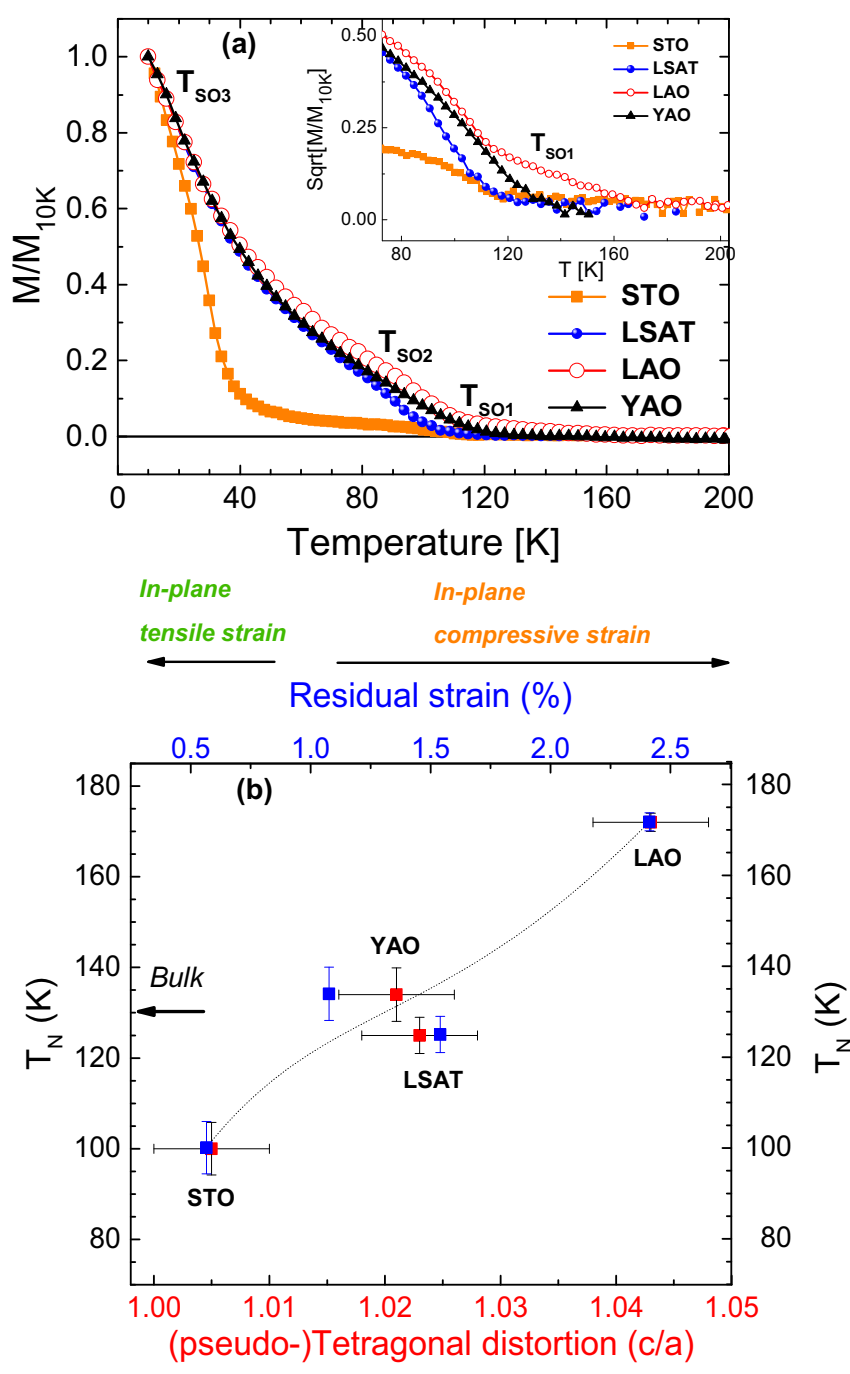

FIG. 4. (a) Normalized-magnetization (ratio of magnetization to the magnetization recorded at $10 \mathrm{~K}$ ) dependence on temperature for PVO films on the YAO, LAO, LSAT, and STO substrates performed at an in-plane applied magnetic field of 50 Oe after field cooling at 5000 Oe. Inset shows the magnified view of MT near $T_{S O 1}$. (b) Variation of the Néel temperature of PVO films with absolute residual strain (top scale) and pseudotetragonal distortion (ratio of out-of-plane to in-plane lattice parameters, c/a) (bottom scale). The closed blue and red squares correspond to the $T_{N}$ plot as a function of actual strain and tetragonal distortion, respectively, along with the error bars. The $T_{N}$ of the bulk PVO is represented by arrow. The dotted line is a guide to the eyes.

TEM measurements) and a small hard component might come from pinning centers due to the microstructure. Figure 3(e) shows variation of the coercivity $\left(H_{c}\right)$ of a hard magnetic phase as a function of the residual strain, whereas $H_{c}$ of a soft phase remains constant at $\sim 0.2 \mathrm{~T}$ for all substrates. The coercivity of hard phase changes from $1.8 \mathrm{~T}$ for a less strained PVO/STO film to 3.6 T for PVO/LAO [Fig. 3(e)]. This is presumably due to an increase of domain wall pinning strength in more strained films.

To understand the presence of soft and hard magnetic phases versus strain, the $M-H$ measurements were performed at different temperatures, i.e., from $10 \mathrm{~K}$ to $100 \mathrm{~K}$.
Interestingly, it was observed that the soft component is present only at temperatures $T<20 \mathrm{~K}$ for PVO/STO and PVO/LSAT but persists up to 80-90 K for PVO/LAO [36]. This indicates the sensitivity of the soft phase for epitaxial strain and temperature and suggests a possible magnetic ordering in PVO films around these temperatures, which triggers the rise of a soft component and will be discussed below.

In order to further investigate the effect of strain on the magnetization of PVO films, the field cooled (FC) and zero field cooled (ZFC) measurements were performed at an inplane applied magnetic field of $H_{\text {in-plane }}=50 \mathrm{Oe}$. For clarity, only FC measurements are shown in this paper with a magnified view near $T_{N}$ (or $T_{S O 1}$ ) [Fig. 4(a)]. The derivative was calculated to visualize the magnetic transitions [36] and results are reported in Table I.

Clearly, a magnetic transition $\left(T_{S O 1}\right)$ is observed for all the films with transition temperature ranging from $100 \mathrm{~K}$ for PVO/STO to $172 \mathrm{~K}$ for PVO/LAO [inset of Fig. 4(a)]. This corresponds to the magnetic transition from a paramagnetic (PM) state to an antiferromagnetic (AFM) phase transition. While for bulk $\mathrm{PrVO}_{3}$ the transition at $T_{S O 1}$ was previously ascribed to the onset of a C-type spin ordering (C-SO) of the canted vanadium moments $[16,41]$, for epitaxial $\mathrm{PrVO}_{3}$ thin films, the substrate-induced strain results in a G-type SO, as evidenced by the DFT calculations, and in agreement with the previous report [21]. More details on G-type SO in PVO films can be found in the Supplemental Material (Sec. 5) [36]. The AFM Néel temperature (named $T_{S O 1}$ here) for $\mathrm{PVO} / \mathrm{STO}$ is however different from our previous report, where $T_{N} \sim 80 \mathrm{~K}$ was reported [32]. This discrepancy could be explained by different growth conditions (especially $P_{\mathrm{O}_{2}}=10^{-5}$ mbar, out-of-plane lattice parameter $=3.97 \AA$ ) which were adopted during deposition. More interesting is the remarkable difference of $\sim 70 \mathrm{~K}$ for the $T_{N}$ of PVO/LAO compared to PVO/STO. Notably, the $M T$ curve also shows two other magnetic features at $T_{\mathrm{SO}_{2}}$ and $T_{\mathrm{SO} 3}$ for LAO, YAO, and LSAT, while the former transition is strongly reduced for STO $\left(T_{\mathrm{SO}_{2}} \sim 30 \mathrm{~K}\right)$. These magnetic orderings were absent in bulk $\mathrm{PrVO}_{3}$ [43], but reported in other orthovanadates of smaller $R$ ionic radii, with decreasing temperature [44-46]. In addition, Reehuis et al. clearly distinguished these transitions for a doped $\operatorname{Pr}_{1-x} \mathrm{Ca}_{x} \mathrm{VO}_{3}$ compound [47]. Upon decreasing the temperature to $T_{\mathrm{SO} 3}$, a slight decrease in magnetization takes place and there is a change in the slope of the magnetizations as well as an anomaly in the inverse susceptibility [36]. This is ascribed to the FM ordering of praseodymium sublattice and/or an AFM coupling between $\operatorname{Pr}^{3+} 4 f$ and $\mathrm{V}^{3+} 3 d$ moments, which results in decrease in the net magnetization below $T_{\mathrm{SO} 3}$. Therefore, by comparing the $\mathrm{MH}$ measurements performed at different temperatures (10-100 K), where a soft component in $M H$ was observed at temperature $T \leqslant 20 \mathrm{~K}$ for $\mathrm{PVO} / \mathrm{STO}$ and PVO/LSAT and up to $80 \mathrm{~K}$ for PVO/LAO [36] and the magnetic transition $T_{S O 3}$ in $M T$, we propose that the soft component in $M H$ results from the AFM coupling between $\mathrm{Pr}$ and $\mathrm{V}^{3+}$ sublattice. For PVO/LAO, the transition at $T_{S O 3}$ in $d M / d T$ is unfortunately difficult to determine as compared to other substrates (Supplemental Material [36], Fig. 7) which exhibit a sharp transition at $T_{\mathrm{SO} 3}$. Additionally, the soft component in $M H$ persists up to $\sim 80 \mathrm{~K}$, meaning that the Pr-V interaction is significantly enhanced for LAO, 
as compared to other substrates. This enhancement is possibly related to the presence of higher strain states in the PVO/LAO film, which raises the degree of Pr-V interaction in the same way as $\mathrm{V}-\mathrm{V}$ interaction $\left(T_{N}\right)$. It is worth noting that another $\mathrm{Pr}^{3+}$ magnetic state may exist at the surface of the PVO film. Indeed, as it has been shown in $\mathrm{DyTiO}_{3}$, thin films that, the over-oxidation at the surface could favor a higher valence state of the transition metal oxide [48]. As a consequence, it would favor $\mathrm{V}^{4+}$ and then alter the exchange interactions with $\mathrm{Pr}$ ions, resulting in isolated paramagnetic $\mathrm{Pr}^{3+}$. Since the measured saturation magnetization remains low compared to $3.57 \mu_{B}$ expected for isolated $\operatorname{Pr}^{3+}$, it seems that the overoxidized surface contribution is rather small. However, the fact that the soft component contribution is modified by changing the substrate indicates rather a modification through the entire film and not only at the surface.

Also, similar to what was earlier reported for bulk $\mathrm{PrVO}_{3}$ [47], the praseodymium sublattice begins to get polarized due to the presence of the exchange field produced by the vanadium sublattice, resulting in a ferrimagnetic structure upon cooling. Here, a small hump at $T \sim 90 \mathrm{~K}$ is also seen, which could be the emergence of another type of spin configuration and/or a phase coexistence between C-SO and G-SO. This seems consistent with the modification of the hysteresis loop as the temperature is lowered through $T_{\mathrm{SO} 2}$, due to switching of spins or change in the spin configuration [36]. However, the feature may be also just related to the overlap of transition regimes $T_{S O 1}$ and $T_{S O 3}$. Further magnetic analysis will be published elsewhere.

To understand further the relationship between the magnetic properties and strain or distortion (ratio of out-of-plane to in-plane lattice parameters), the $T_{N}$ versus lattice strain is plotted for the PVO films, as shown in Fig. 4(b). The $T_{N}\left(T_{S O 1}\right)$ of the PVO films increases altogether with the residual strain, which is highest for LAO $\left(T_{N} \sim 172 \mathrm{~K}\right)$ and lowest for STO $\left(T_{N} \sim 100 \mathrm{~K}\right)$, while PVO film on YAO has $T_{N}$ close to the bulk PVO $\left(T_{N} \sim 130 \mathrm{~K}\right)$. Furthermore, the PVO/YAO film is in-plane fully relaxed while the out-of-plane lattice parameter is larger than the bulk. This produces a distorted structure with $c / a$ ratio $\sim 1.02$. The enhancement of the out-of-plane lattice parameter of PVO/YAO might be a result of defects in film such as oxygen vacancies, etc. It is interesting to note that the influence of small compressive strain (LSAT) in PVO film is similar to bulk, where a small tensile strain (STO) decreases the $T_{N}$ by $30 \mathrm{~K}$ [43]. On the other hand, it requires a large compressive strain of $2.4 \%$ (LAO) to increase the same by $40 \mathrm{~K}$; cf. bulk.

To further explore the magnetic properties of PVO films and their dependence on strain, which lead to a tilting of $\mathrm{BO}_{6}$ octahedra or change in the B-O-B bond angle [49-53], it is necessary to have a complete knowledge of distortion of the structure and the $\mathrm{VO}_{6}$-octahedral rotation. From previous studies of strained oxide perovskites, the degree of rotation of $\mathrm{BO}_{6}$ octahedra depends strongly on sign and the magnitude of the strain [52,53]. Under tensile in-plane strain $(c / a<1.01)$, the $\mathrm{VO}_{6}$ octahedra are comprised of an enhanced in-plane $\mathrm{V}-\mathrm{O}$ bond length and $\mathrm{V}-\mathrm{O}-\mathrm{V}$ bond angle close to $180^{\circ}$. This decreases the in-plane AFM superexchange interaction between nearest neighbor sites, hence reducing $T_{N}$. On the other hand, under compressive in-plane strain $(c / a<1.01)$, it is the other way around, i.e., a reduced in-plane $\mathrm{V}-\mathrm{O}$ bond length and $\mathrm{V}-\mathrm{O}-\mathrm{V}$ bond angle $<180^{\circ}$. This, as a result, enhances the in-plane AFM interaction and therefore enhanced $T_{N}$.

\section{FIRST-PRINCIPLES SIMULATIONS}

To get further insights on the role of the epitaxial strain on the magnetic properties of $\mathrm{PrVO}_{3}$ films, we have performed first-principles simulations using density functional theory (DFT). Consistent with previous studies [21], DFT correctly predicts that bulk $\mathrm{PrVO}_{3}$ is a $\mathrm{C}$-SO insulator in the ground state. Regarding the thin films, we find that the perovskite grows with the (001) and (1-10) Pbnm axes aligned along the substrate for all the tested films [ e.g., $\mathrm{PrVO}_{3}$ grown on STO, LSAT, LAO, and YAO substrates; see insets of Fig. 5(b) for sketches of local axes and growth orientation]. This yields films grown along the orthorhombic (110) direction, in sharp agreement with experiments. We emphasize here that due to the presence of small domains in the films inducing a mechanical constraint [21], we have considered growth conditions with the $(110)_{o}$ direction forced to be orthogonal to the substrate (i.e., the film is not allowed to tilt). With that additional constraint, the ground state is associated with a $P 2_{1} / m$ symmetry with nearest neighbor $\mathrm{V}^{3+}$ spins antiferromagnetically coupled in all crystallographic directions. It yields a G-type spin ordering compatible with experiments. Finally, all films are insulating with band gaps ranging from $1.50 \mathrm{eV}$ (YAO) to $1.78 \mathrm{eV}$ (STO).

Although mean-field methods such as DFT cannot provide accurate values of the Néel temperature, they nevertheless remain valuable techniques for capturing trends as a function of external stimuli [54]. We report on Fig. 5(a) the ratio of the Néel temperature with respect to that of $\mathrm{PrVO}_{3}$ grown on a STO substrate as a function of the pseudotetragonality $c / a$ of the films extracted from our simulations (see method for details on evaluation of the Néel temperature). As one can see, DFT captures the trend observed experimentally with an enhancement of the Néel temperature going from STO to YAO substrates, although our computed $T_{N} / T_{N \text {-STO }}$ ratio is smaller than the experimental one for the LAO substrate. Amazingly, if the material could be stabilized on YAO without relaxation of the film, the Néel temperature is expected to be approximately multiplied by two with respect to that of $\mathrm{PrVO}_{3}$ films deposited on STO.

Along with validating the experimentally measured trend for $T_{N}$ as a function of the applied epitaxial strain, our firstprinciples simulations also provide microscopic insights on the origin of this physical behavior. We observe that both magnetic constants $J_{1}$ and $J_{2}$ between nearest $\mathrm{V}^{3+}$ neighbors along the (1-10) [or (110)] and (001) directions, respectively, increase with enlarging the compressive epitaxial strain. First, this is ascribed to shorter $\mathrm{V}-\mathrm{O}$ bond lengths along the (1-10) and (001) directions induced by strain. Second, we do not observe any significant modifications of oxygen cage rotation amplitude in the different films- the $a^{-} a^{-} c^{0}$ rotation amplitude is even slightly increasing with decreasing the substrate lattice parameter-and thus the classical " $\mathrm{V}-\mathrm{O}-\mathrm{V}$ angles going to $180^{\circ}$ " argument cannot explain the strengthening of $J$. Nevertheless, we find a crossover between two lattice distortions as a function of the epitaxial strain [see Fig. 5(b)]: 

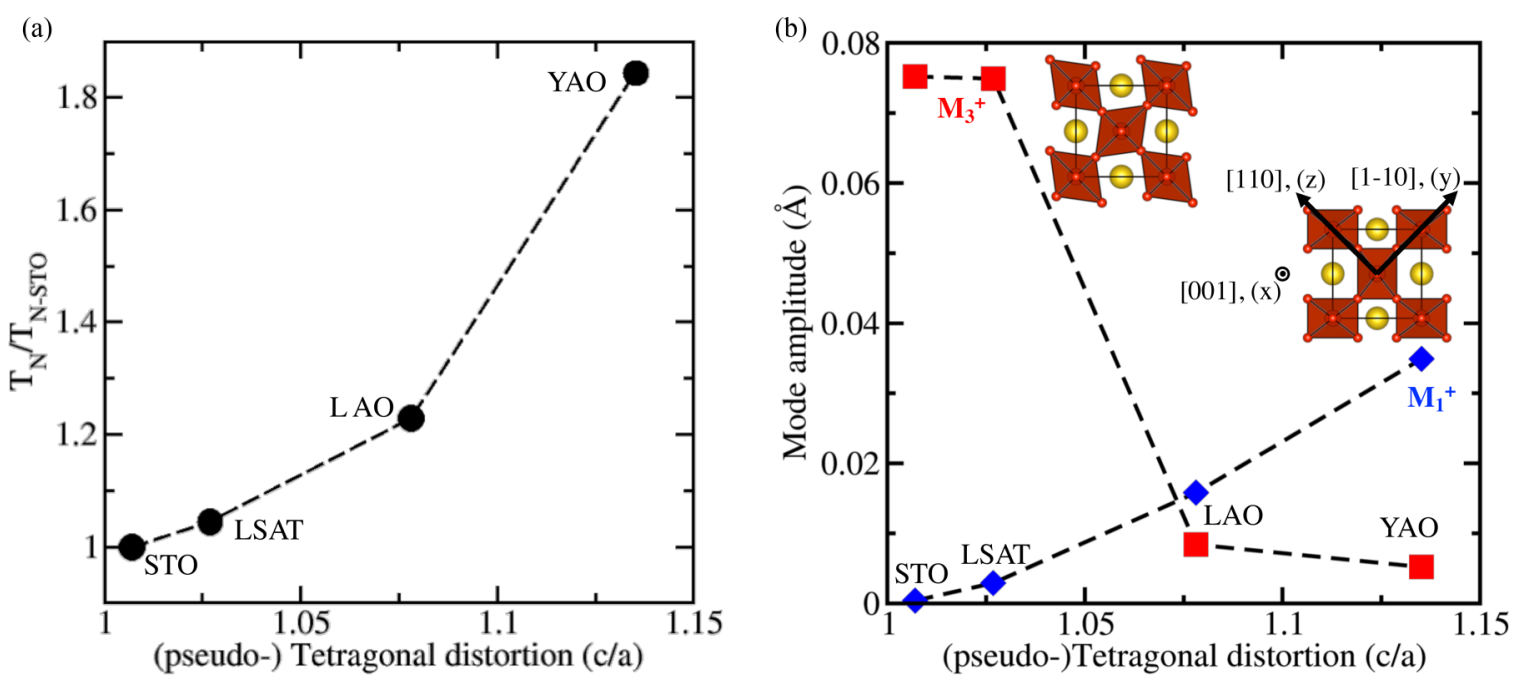

FIG. 5. Relative evolution of the Néel temperature (a) and of the amplitude of the $\mathbf{M}_{3}^{+}$and $\mathbf{M}_{1}^{+}$distortions as a function of the pseudotetragonality of the $\mathrm{PrVO}_{3}$ films. Sketches of the structural distortions and local axes are presented as insets.

(i) for a moderate lattice mismatch ( e.g., STO and LSAT), we extract a large Jahn-Teller distortion, labeled $\mathrm{M}_{3}^{+}$, producing an asymmetry of $\mathrm{V}-\mathrm{O}$ bonds on nearest $\mathrm{V}$ sites that is reminiscent of bulk $\mathrm{RVO}_{3}$ physics and (ii) for a large lattice mismatch (e.g., LAO and YAO), the JT motion vanishes and is replaced by a $\mathrm{M}_{1}^{+}$distortion unaffecting $\mathrm{V}-\mathrm{O}$ bond lengths but distorting $\mathrm{O}-\mathrm{V}-\mathrm{O}$ angles in $\mathrm{VO}_{2}$ planes orthogonal to the (001) direction [see insets of Fig. 5(b) for sketches of the distortions]. The amplitude of the latter distortion, absent in the bulk and roughly zero for films grown on STO and LSAT substrates, closely behaves like $T_{N}$ as a function of the tetragonality of the material. In fact, the crossover between the amplitude associated with the $\mathrm{M}_{1}^{+}$and $\mathrm{M}_{3}^{+}$distortions highlights a clear modification of the electronic structure: the two $\mathrm{V}^{3+} d$ electrons are located in the $d_{y z}$ orbital plus an alternating combination of the $d_{x z} \pm d_{x y}$ orbitals on neighboring sites for moderate strains, while they lie in the $d_{x z}$ and $d_{y z}$ orbitals on all neighboring sites for large compressive epitaxial strain [see insets of Fig. 5(b) for the definition of local axes]. It follows that $\mathrm{V}-\mathrm{O}$ bond length contractions combined with the modifications of $\mathrm{V}^{3+} d$ orbital occupancies for $\mathrm{LAO}$ and YAO substrates favor superexchange in the three crystallographic directions and thus strongly promote the enhancement of the Néel temperature.

This illustrates that not only cooperative octahedral-site rotation, i.e., rigid octahedra tilts and rotations, may tune the physical properties but also octahedral-site distortion through electronic state modifications as reported for bulk orthorhombic perovskite $[55,56]$. We show here that octahedral-site distortion can be driven by mechanical strain engineering and should be considered for other epitaxial orthorhombic perovskite thin films.

\section{CONCLUSION}

In conclusion, we have successfully grown single-phased $\mathrm{PrVO}_{3}$ thin films on top of various single crystal substrates.
The most distorted structure with $c / a \sim 1.04$ is observed on the LAO substrate where a large strain of $2.4 \%$ is measured. Furthermore, a relationship between the magnetic properties and the structural distortion $(c / a)$ in $\mathrm{PrVO}_{3}$ films was developed. We have also evidenced a clear ferromagnetic behavior of $\mathrm{PrVO}_{3}$ thin films at low temperature and shown that the $M H$ hysteresis loop is comprised of two magnetic sublattices, which gives rise to a soft and a hard ferromagneticlike component in $M H$. The magnetic phase diagram $\left(T_{N}\right.$ vs $\left.c / a\right)$ for $\mathrm{PrVO}_{3}$ films was mapped out for $1<c / a<1.04$. The most distorted film has $T_{N} \sim 172 \mathrm{~K}, 40 \mathrm{~K}$ higher than the bulk, whereas the least distorted film has $T_{N} \sim 100 \mathrm{~K}, 30 \mathrm{~K}$ lower than bulk, making PVO films an eligible candidate for an application point of view for wide range tuning of its magnetic transition temperature. Finally, the first-principles simulations have confirmed that the compressive strain not only produces stronger magnetic interactions, but also promotes electronic states totally absent of the bulk.

\section{ACKNOWLEDGMENTS}

The authors thank F. Veillon for his valuable experimental support. This work is supported by Region Normandie and by french ANR POLYNASH and Labex EMC3. C.U.J. thanks Université de Caen Normandie for support through his visiting fellowship. B.D. acknowledges the financial support of the program EQUIPEX GENESIS, Agence Nationale de la Recherche (Grant No. ANR-11-EQPX-0020) for TEM lamella preparation. Ph.G. acknowledges the F.R.S/F.N.R.S PDR project HiT4FiT and ARC project AIMED. Firstprinciples calculations were performed at Abel supercomputers through the PRACE project TheoMoMuLaM and at the Cartesius supercomputer through the PRACE Project TheDeNoMO. The authors also took advantage of the Céci-HPC facilities funded by F.R.S.-FNRS (Grant No. 2.5020.1) and the Tier-1 supercomputer of the Fédération Wallonie-Bruxelles funded by the Walloon Region (Grant No. 1117545). 
[1] M. Sayer, P. Chen, R. Fletcher, and A. Mansingh, J. Phys. C 8, 2059 (1975).

[2] H. Meley, Karandeep, L. Oberson, J. de Bruijckere, D. T. L. Alexander, J.-M. Triscone, P. Ghosez, and S. Gariglio, APL Mater. 6, 046102 (2018).

[3] S.-P. Matsuda, S. Takeuchi, A. Soeta, T. Doi, K. Aihara, and T. Kamo, Jpn. J. Appl. Phys. 29, L1781 (1990).

[4] N. F. Mott, Metal-Insulator Transitions (Taylor and Francis, London, 1990).

[5] P. Zubko, S. Gariglio, M. Gabay, P. Ghosez, and J.-M. Triscone, Annu. Rev. Condens. Matter Phys. 2, 141 (2011).

[6] J. H. Haeni, P. Irvin, W. Chang, R. Uecker, P. Reiche, Y. L. Li, S. Choudhury, W. Tian, M. E. Hawley, B. Craigo, A. K. Tagantsev, X. Q. Pan, S. K. Streiffer, L. Q. Chen, S. W. Kirchoefer, J. Levy, and D. G. Schlom, Nature (London) 430, 758 (2004).

[7] D. Sando, A. Barthélémy, and M. Bibes, J. Phys.: Condens. Matter 26, 473201 (2014).

[8] M. Čulo, M. Basletić, E. Tafra, A. Hamzić, S. Tomić, F. Fischgrabe, V. Moshnyaga, and B. Korin-Hamzić, Thin Solid Films 631, 205 (2017).

[9] J. Fontcuberta, C. R. Phys. 16, 204 (2015).

[10] F. Yen, C. dela Cruz, B. Lorenz, E. Galstyan, Y. Y. Sun, M. Gospodinov, and C. W. Chu, J. Mater. Res. 22, 2163 (2007).

[11] N. Aliouane, O. Prokhnenko, R. Feyerherm, M. Mostovoy, J. Strempfer, K. Habicht, K. C. Rule, E. Dudzik, A. U. B. Wolter, A. Maljuk, and D. N. Argyriou, J. Phys.: Condens. Matter 20, 434215 (2008).

[12] T. Katsufuji, M. Masaki, A. Machida, M. Moritomo, K. Kato, E. Nishibori, M. Takata, M. Sakata, K. Ohoyama, K. Kitazawa, and H. Takagi, Phys. Rev. B 66, 134434 (2002).

[13] J. Fujioka, T. Yasue, S. Miyasaka, Y. Yamasaki, T. Arima, H. Sagayama, T. Inami, K. Ishii, and Y. Tokura, Phys. Rev. B 82, 144425 (2010).

[14] J. F. Mitchell, D. N. Argyriou, A. Berger, K. E. Gray, R. Osborn, and U. Welp, J. Phys. Chem. B 105, 10731 (2001).

[15] J. Varignon, M. Bibes, and A. Zunger, Nat. Commun. 10, 1658 (2019).

[16] M. H. Sage, G. R. Blake, C. Marquina, and T. T. M. Palstra, Phys. Rev. B 76, 195102 (2007).

[17] K. Kugel and D. Khomskii, Zh. Eksp. Teor. Fiz. 64, 1429 (1973).

[18] J. B. Goodenough, Magnetism and Chemical Bond Interscience (New York, John Wiley and sons 1963).

[19] J.-S. Zhou, J. B. Goodenough, J.-Q. Yan, and Y. Ren, Phys. Rev. Lett. 99, 156401 (2007).

[20] D. Bizen, K. Nakatsuka, T. Murata, H. Nakao, Y. Murakami, S. Miyasaka, and Y. Tokura, Phys. Rev. B 78, 224104 (2008).

[21] O. Copie, J. Varignon, H. Rotella, G. Steciuk, P. Boullay, A. Pautrat, A. David, B. Mercey, P. Ghosez, and W. Prellier, Adv. Mater. 29, 1604112 (2017).

[22] G. Kresse and J. Hafner, Phys. Rev. B 47, 558 (1993).

[23] G. Kresse and J. Furthmiiller, Comput. Mater. Sci. 6, 15 (1996).

[24] S. L. Dudarev, G. A. Botton, S. Y. Savrasov, C. J. Humphreys, and A. P. Sutton, Phys. Rev. B 57, 1505 (1998).

[25] J. Varignon, N. C. Bristowe, E. Bousquet, and P. Ghosez, Sci. Rep. 5, 15364 (2015).

[26] P. E. Blöchl, Phys. Rev. B 50, 17953 (1994).
[27] G. W. Watson, S. C. Parker, and G. Kresse, Phys. Rev. B 59, 8481 (1999).

[28] A. Biswas and Y. H. Jeong, J. Appl. Phys. 117, 195305 (2015).

[29] R. D. King-Smith and D. Vanderbilt, Phys. Rev. B 49, 5828 (1994).

[30] O. Diéguez, K. M. Rabe, and D. Vanderbilt, Phys. Rev. B 72, 144101 (2005).

[31] G. Herranz, M. Basletić, O. Copie, M. Bibes, A. N. Khodan, C. Carrétéro, E. Tafra, E. Jacquet, K. Bouzehouane, A. Hamzić, and A. Barthélémy, Appl. Phys. Lett. 94, 012113 (2009).

[32] O. Copie, H. Rotella, P. Boullay, M. Morales, A. Pautrat, P.-E. Janolin, I. C. Infante, D. Pravathana, U. Lüders, and W. Prellier, J. Phys.: Condens. Matter 25, 492201 (2013).

[33] J. A. Moyer, C. Eaton, and R. Engel-Herbert, Adv. Mater. 25, 3578 (2013).

[34] U. Aschauer, R. Pfenninger, S. M. Selbach, T. Grande, and N. A. Spaldin, Phys. Rev. B 88, 054111 (2013).

[35] P. Agrawal, J. Guo, P. Yu, C. Hébert, D. Passerone, R. Erni, and M. D. Rossell, Phys. Rev. B 94, 104101 (2016).

[36] See Supplemental Material at http://link.aps.org/supplemental/ 10.1103/PhysRevB.99.224405 for details of film thickness simulation, a comparative summary of TEM observations, strain effect on the weightage of soft and hard magnetic component, and derivative of $M T, \mathrm{Pr}-\mathrm{V}$ interaction.

[37] D. Kumar, A. David, A. Fouchet, B. Domengès, and W. Prellier (unpublished).

[38] H. Rotella, O. Copie, A. Pautrat, P. Boullay, A. David, D. Pelloquin, C. Labbé, C. Frilay, and W. Prellier, J. Phys.: Condens. Matter 27, 095603 (2015).

[39] H. P. R. Frederikse and W. R. Hosler, Phys. Rev. 161, 822 (1967).

[40] L. D. Tung, Phys. Rev. B 72, 054414 (2005).

[41] F. Wang, J. Zhang, P. Yuan, Q. Yan, and P. Zhang, J. Phys.: Condens. Matter 12, 3037 (2000).

[42] J. Scola, P. Boullay, W. Noun, E. Popova, Y. Dumont, A. Fouchet, and N. Keller, J. Appl. Phys. 110, 043928 (2011).

[43] S. Miyasaka, Y. Okimoto, M. Iwama, and Y. Tokura, Phys. Rev. B 68, 100406(R) (2003).

[44] T. Sarkar, S. A. Ivanov, G. V. Bazuev, P. Nordblad, and R. Mathieu, J. Phys. D 48, 345003 (2015).

[45] T. Sakai, G. Adachi, J. Shiokawa, and T. Shinike, J. Appl. Phys. 48, 379 (1977).

[46] Q. Zhang, K. Singh, C. Simon, L. D. Tung, G. Balakrishnan, and V. Hardy, Phys. Rev. B 90, 024418 (2014).

[47] M. Reehuis, C. Ulrich, P. M. Abdala, P. Pattison, G. Khaliullin, J. Fujioka, S. Miyasaka, Y. Tokura, and B. Keimer, Phys. Rev. B 94, 104436 (2016).

[48] R. Aeschlimann, D. Preziosi, P. Scheiderer, M. Sing, S. Valencia, J. Santamaria, C. Luo, H. Ryll, F. Radu, R. Claessen, C. Piamonteze, and M. Bibes, Adv. Mater. 30, 1707489 (2018).

[49] A. Y. Borisevich, H. J. Chang, M. Huijben, M. P. Oxley, S. Okamoto, M. K. Niranjan, J. D. Burton, E. Y. Tsymbal, Y. H. Chu, P. Yu, R. Ramesh, S. V. Kalinin, and S. J. Pennycook, Phys. Rev. Lett. 105, 087204 (2010).

[50] H. Rotella, U. Lüders, P.-E. Janolin, V. H. Dao, D. Chateigner, R. Feyerherm, E. Dudzik, and W. Prellier, Phys. Rev. B 85, 184101 (2012). 
[51] J. M. Rondinelli and N. A. Spaldin, Phys. Rev. B 82, 113402 (2010).

[52] S. J. May, J.-W. Kim, J. M. Rondinelli, E. Karapetrova, N. A. Spaldin, A. Bhattacharya, and P. J. Ryan, Phys. Rev. B 82, 014110 (2010).

[53] A. Vailionis, H. Boschker, W. Siemons, E. P. Houwman, D. H. A. Blank, G. Rijnders, and G. Koster, Phys. Rev. B 83, 064101 (2011).
[54] D. M. Korotin, V. V. Mazurenko, V. I. Anisimov, and S. V. Streltsov, Phys. Rev. B 91, 224405 (2015).

[55] J.-S. Zhou and J. B. Goodenough, Phys. Rev. B 77, 132104 (2008).

[56] J.-S. Zhou, J. A. Alonso, V. Pomjakushin, J. B. Goodenough, Y. Ren, J.-Q. Yan, and J.-G. Cheng, Phys. Rev. B 81, 214115 (2010). 